\title{
Enabling Interactive Analytics of Secure Data using Cloud Kotta
}

\author{
Yadu N. Babuji, Kyle Chard, and Eamon Duede \\ Computation Institute, University of Chicago and Argonne National Laboratory \\ 5735 S Ellis Ave \\ Chicago, Illinois 60637 \\ \{yadu,chard,eduede\}@uchicago.edu
}

\begin{abstract}
Research, especially in the social sciences and humanities, is increasingly reliant on the application of data science methods to analyze large amounts of (often private) data. Secure data enclaves provide a solution for managing and analyzing private data. However, such enclaves do not readily support discovery science-a form of exploratory or interactive analysis by which researchers execute a range of (sometimes large) analyses in an iterative and collaborative manner. The batch computing model offered by many data enclaves is well suited to executing large compute tasks; however it is far from ideal for day-to-day discovery science. As researchers must submit jobs to queues and wait for results, the high latencies inherent in queue-based, batch computing systems hinder interactive analysis. In this paper we describe how we have augmented the Cloud Kotta secure data enclave to support collaborative and interactive analysis of sensitive data. Our model uses Jupyter notebooks as a flexible analysis environment and Python language constructs to support the execution of arbitrary functions on private data within this secure framework.
\end{abstract}

\section{ACM Reference format:}

Yadu N. Babuji, Kyle Chard, and Eamon Duede. 2017. Enabling Interactive Analytics of Secure Data using Cloud Kotta. In Proceedings of Workshop on Scientific Cloud Computing, Washington, DC USA, June 2017 (ScienceCloud'17), 6 pages.

DOI: $10.475 / 123 \_4$

\section{INTRODUCTION}

Regardless of domain, scientists are rapidly embracing data-driven science as a means of extracting knowledge from large amounts of data. While there are many examples of successful research based upon big data in the biomedical [15], physical [12] and social [10] sciences, many researchers still face challenges managing and analyzing large amounts of data. These challenges are even more complex when the data to be analyzed is sensitive, private, or valuable. Furthermore, the increasingly common adoption of 'discovery science', an approach that centers on iterative and exploratory analysis of large volumes of data to discover patterns and correlations, is beyond the reach of many researchers who lack the expertise and computational infrastructure to support such an approach to interrogating large, sensitive data. In practice, the requirements for analyzing sensitive, big data using discovery and

Permission to make digital or hard copies of part or all of this work for personal or classroom use is granted without fee provided that copies are not made or distributed for profit or commercial advantage and that copies bear this notice and the full citation on the first page. Copyrights for third-party components of this work must be honored. For all other uses, contact the owner/author(s).

ScienceCloud'17, Washington, DC USA

(c) 2017 Copyright held by the owner/author(s). $123-4567-24-567 / 08 / 06 \ldots \$ 15.00$ DOI: $10.475 / 123 \_4$ data science methodologies necessitates flexible, intuitive, and efficient infrastructure. Yet, most highly secure, big data computational infrastructure is anything but flexible and intuitive.

To address the need for the secure and scalable management and analysis of data, we developed the Cloud Kotta secure data enclave [6, 7] Cloud Kotta provides a cloud-based, elastically scalable environment that is able to meet the needs of sporadic and bursty scientific analysis workloads while removing the need for owning and operating large scale infrastructure. It implements a fine grain access control model over managed research data allowing controlled access from within and outside the enclave. To address the need for reliability, scalability and collaborative access, Cloud Kotta is built upon Amazon Web Services (AWS). Over the past year, Cloud Kotta has been leveraged by dozens of researchers and students to analyze data using more than a quarter million core hours.

While Cloud Kotta has shown immense value, it, like many other data enclaves, offers only a queue-based job submission model. Unfortunately, such models are not well-suited to the increasingly common discovery science approaches used by researchers. Rather, discovery and data science approaches typically rely on lightweight scripting languages (e.g., $\mathrm{R}$ and Python), flexible data structures (e.g., dataframes), inline visualizations (to 'inspect' the data), and exploratory statistical and machine learning algorithms. These days, researchers rely on interactive analysis environments like Jupyter Notebooks [2] to support iterative and collaborative analysis that marries code, equations, documentation, results, and visualizations. A quick and responsive environment that allows for fast, iterative development is an ideal fit for analysis and discovery tasks. For this reason, these interactive development environments have quickly become crucial tools of the applied data science community and are starting to gain favor across a broad range of computational sciences. However, maintaining the high availability requirements of interactive compute resources can be expensive especially with a large group of researches to support. Furthermore, providing interactive analysis of secure data is particularly challenging.

In this paper, we describe a model for enabling interactive, multiuser analysis of secure data. We base our model on Jupyter Notebooks and Cloud Kotta to provide the security of a data enclave, scalable compute, and the interactivity required of today's discovery science. We have developed a Python library that enables specific functions in an analysis code, written in a Jupyter Notebook, to be seamlessly and securely submitted to the Cloud Kotta execution fabric. Our approach streamlines the interfaces between the analysis code and the execution environment thereby offering native, synchronous Python input/output alongside an asynchronous jobbased model for long running analyses. To validate our model we 
describe several real-world use cases for which this system was used.

\section{BACKGROUND AND MOTIVATION}

A gap has opened up between researchers' preferred methods for working with and interrogating data and the usability of the computational environments that host that work. Researchers are increasingly adopting highly flexible, iterative, approaches to data exploration. These approaches are most prominent in the applied data science and discovery science regimes in which large quantities of heterogeneous data are explored, smashed together, interrogated by general and specialized data mining and machine learning tools in a highly iterative process of rapid ideation and exploration. As this methodology has gained favor, researchers have attempted to leverage it in working with ever larger, more diverse, and highly sensitive data (e.g., patient, genetic, tax, commercial, etc.). At the same time, computational facilities, resources, and platforms have sought to optimize throughput, performance, and security with little regard for the interactivity or the intuitive, inventive, flights of exploratory fancy that researchers now crave. On one side of the gap, users of these facilities now have scale at the expense of flexibility and security at the expense of interactivity. On the other, users have flexibility and interactivity at the expense of scale and security.

Cloud Kotta was developed to serve the computational needs of a diverse network of scientists that rely on big data and computation to ask and answer big questions. Originally, Cloud Kotta was designed to virtually centralize the research efforts of a decentralized group around highly protected datasets. In order to carry out analysis of highly sensitive data at a distance, researchers were (initially) willing to suffer shortcomings related to interactivity and flexibility. However, iterating on models and troubleshooting bugs in the fairly rigid, queue-based job submission analysis workflows that Cloud Kotta supported proved cumbersome and, ultimately, frustrated research efforts.

For this reason, we sought to extend Cloud Kotta by developing and providing an interactive analysis environment that enables users to work directly with elastic computational resources and highly sensitive data in a flexible, secure, and interactive environment at scale. Specifically, for interactive data analysis on Cloud Kotta to be efficacious, we derived the following requirements.

The augmented system needed to: 1) maintain high levels of security, ensuring that only permitted users are able to access and interact with data while restricting what data can leave the system; 2) provide an intuitive interface that is both familiar to users and flexible enough to support a broad range of analysis types; 3 ) seamlessly integrate with a diverse set of programming languages so that users do not need to make significant modifications to their programs; 4) scale to support very large amounts of data and use of large compute resources; and 5) embrace software as a service models such that users can access these capabilities without installing software locally.

In what follows, we sketch the Cloud Kotta architecture and then describe in detail our novel Jupyter integrations that enable interactive analysis of data within the Cloud Kotta environment.

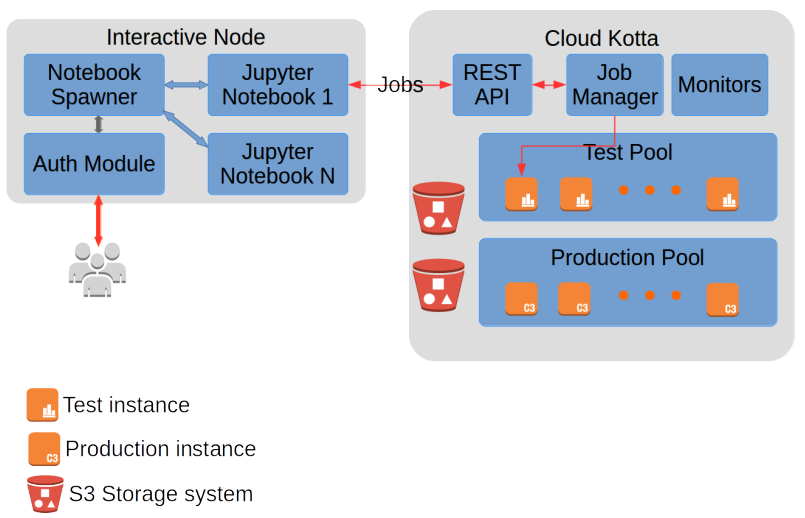

Figure 1: Architecture.

Additionally, we discuss three real-world use cases that have leveraged these capabilities to conduct science.

\section{DESIGN AND ARCHITECTURE}

In an effort to address the requirements described in the previous section, we extended Cloud Kotta to integrate the Jupyter development environment and marry its interactive execution model with the Cloud Kotta computation fabric. Our approach allows users to select and analyze secure datasets while leveraging the virtually infinite compute and storage capacity of the cloud all within a familiar and intuitive environment. The architecture of our solution is depicted in Figure 1.

\subsection{Cloud Kotta}

Cloud Kotta is a cloud-based service for conducting collaborative research around protected datasets. It is designed to be deployed on Amazon Web Services (AWS), leveraging highly reliable platform services and scalable data storage and compute environments. Cloud Kotta leverages a suite of AWS service including Simple Storage Service (S3) for long term data storage, DynamoDB for storing fine grain job histories, Simple Queue Service (SQS) for rapid and reliable task distribution, and Elastic Compute Cloud (EC2) for an elastically scalable computing environment.

Cloud Kotta stores and manages protected data in S3. This provides a low cost model for storing large amounts of data while also providing a fine grain access control model for individual objects. Moreover, Kotta offers secure HTTPS interfaces for accessing data. When data is to be analyzed, it is moved and cached on cloud instance storage using AWS Elastic Block Storage. Cloud Kotta employs advanced data management policies that move data within storage tiers based on frequency of access. This model allows us to trade off high availability for lower cost similar to least-recentlyused caching. Combining these methods allows Cloud Kotta to reduce storage costs, significantly.

To satisfy bursty and potentially large-scale compute workloads Cloud Kotta employs multiple elastic pools of compute resources. This allows the system to match incoming workloads so as to provide seemingly infinite compute capacity at a fraction of the cost of maintaining a large persistent cluster in the cloud. That is, instances are only initiated when needed so that users are only charged for the resources needed for compute. Where possible, Cloud Kotta 
leverages low cost spot instances and adopts a smart bidding system across four availability zones to ensure that the resources provisioned are the cheapest available machines.

To service the two broad job categories on the system, Cloud Kotta maintains a test queue that is geared for development jobs and a separate production queue for jobs that require significant $\mathrm{CPU}$ and or memory resources. The test queue is comprised of an elastic pool of t2.medium instances, each with $2 \mathrm{vCPUs}$ and $4 \mathrm{~GB}$ of RAM. These instances are on-demand instances which can be provisioned in under two minutes. To ensure rapid response time, the test queue always has at least one machine on-line. The production queue, on the other hand, is usually populated with c3.8xlarge (32vCPU, 60GB RAM) or i2.8xlarge (36vCPU, 244GB RAM) machines that are provisioned from the spot market. These machines are slower to provision and are designed for jobs that are generally tolerant of delays.

In order to utilize these resources, Cloud Kotta provides a queue based job submission model that is accessible through a Web GUI, REST API and a command line interface. The Web GUI is convenient for submitting single tasks and tracking their progress, but become a hassle when dealing with a large number of jobs. The REST interface is designed to support programmatic access for example from external applications of via scripts that aim to manage several hundreds of jobs. This flexibility comes with the additional effort of fitting science workflows to this model. The command line interface is useful for submitting a large number of jobs with minor variations. This is suited for bash script based tasks.

\subsection{Security}

Security of the hosted data is the primary concern for a secure data enclave. Cloud Kotta is architected with this in mind, and so implements a rich, fine grain access control model. At the heart of the model users are assigned roles. All datasets define policies with respect to these roles, for example defining who can access each dataset. Policies also control what permissions individual services have. Every request that involves data is signed and logged such that access can be audited in the future.

Rather than implement a user management system, Cloud Kotta leverages Amazon's identity services. Cloud Kotta's web interface relies on Login with Amazon, an OAuth 2.0 provider for user authentication. This workflow allows users to authenticate with their Amazon identity. Cloud Kotta is given a short-term token that can be used to verify identity and conduct operations on that user's behalf. While the authentication tokens themselves are valid for only an hour, we create cookies to extend session validity to 6 hours. This approach works well for web-based sessions; however it is not suitable in a programmatic context. To simplify programmatic authentication Cloud Kotta leverages refresh tokens issued through a one-time registration process. These tokens can be refreshed by the application for as long as they are valid. Users may revoke tokens at any point in the future.

To support analysis of private data on elastic cloud instances Cloud Kotta leverages a unique delegated access model. Each instance is created with a special role, this role provides a minimal set of privileges by default (e.g., to read from the queue). However, the role allows the instance to inherent the role of the user that owns a submitted job. In this case, the instance changes to the user's role which allows it to access protected data throughout the execution of the analysis task.

Cloud Kotta applies best practices cloud security models to secure the entire system. For example, S3 buckets are encrypted and accessible only from within a predefined virtual private cloud (VPC). The compute layer is isolated from the internet by a private subnet.

\subsection{Jupyter Deployment}

Jupyter is an application that allows users to author living documents that contain code (e.g., Python, R, Julia), rich descriptive text (e.g., documentation), and the results of running code (e.g., text, figures). Notebooks are organized into cells, users can execute these cells in any order and state is shared between cells. Jupyter notebooks are authored and executed in a web browser, using a locally deployed web server. One of the reasons Jupyter has become so popular is that it provides a flexible and interactive model for analysis, it is intuitive to use via a web browser, and notebooks are self-describing. It is this combination that makes it easy to share, reproduce, and extend analyses. However, Jupyter notebooks are limited in that they are single-user instances, they are not designed to exploit large scale computing infrastructure, and they do not provide support for accessing data securely.

JupyterHub [3] is a multi-user server that manages user authentication and can spawn multiple single-user Jupyter notebooks. We base our integration on JupyterHub for this reason. To do so, we have deployed JupyterHub on an EC2 instance within the Cloud Kotta system. We use JupyterHub's PAM authentication system to authenticate users onto the login node with access to a persistent filesystem. This authentication system is currently separate from the system used by Cloud Kotta and therefore requires a second user account. We are actively working to integrate the two models. JupyterHub users are provided with their own (isolated) space that can be used for creating and maintaining stateful development environments (e.g., notebooks and temporary data). We use a notebook spawner to create new notebook instances for users on demand. This spawner is designed to restrict the CPU and RAM available to each notebook such that individual users will not be able to accidentally render the service unusable. As we will describe later, our library extensions allow users to offload analysis tasks that require resources beyond what is allocated to an individual notebook instance.

Since availability and reliability is critical for the Jupyter environment, it is important that the host EC2 instance capacity matches expected workload (i.e., number of users). To address this concern, the Cloud Kotta system administration team monitors load and can scale-up or scale-down the instance type that is provisioned for the service.

\subsection{Kotta Interactive Library}

The primary interface used to connect Jupyter notebooks with Cloud Kotta is the kotta python library. This library enables the user to markup python functions using a decorator. Functions decorated with the @kottajob decorator, when called are executed transparently on the Cloud Kotta infrastructure. The library handles the serialization of the arguments passed, canning of the decorated 


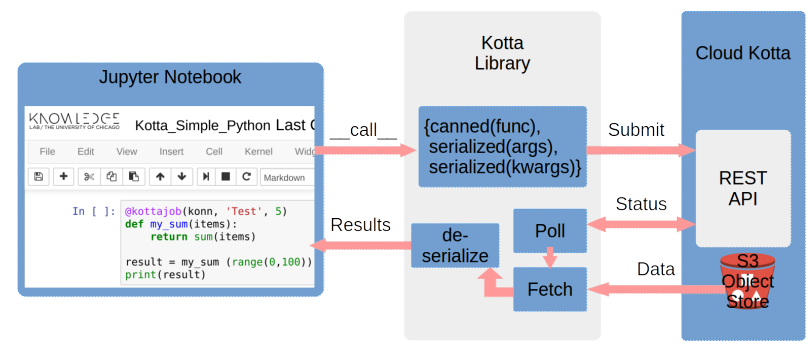

Figure 2: Kotta Library sequence diagram

function, and deserialization of the results once they have been computed. The decorator takes an authenticated connection object, the target Cloud Kotta queue and a walltime as required arguments. The kotta library authenticates with Cloud Kotta using valid access tokens. These tokens are, at present, stored in a file within the user's private file system. This sequence of steps taken by the kotta library is shown in Figure 2

Here is a simple example of a decorated Python function which simply computes the sum of a list of integers:

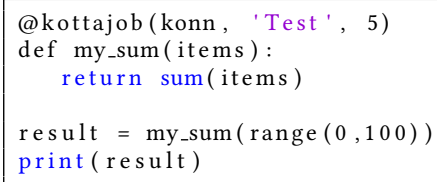

The above code snippet, when executed with the kotta library, looks to the user like any other Python script, albeit with the additional latencies from serialization, transmission and execution on a remote compute node. The function runs in blocking mode, therefore my_sum() does not return until its results are available. The akottajob decorator can also run in non-blocking mode and that makes it simple to exploit many-task parallelism within analysis workflows.

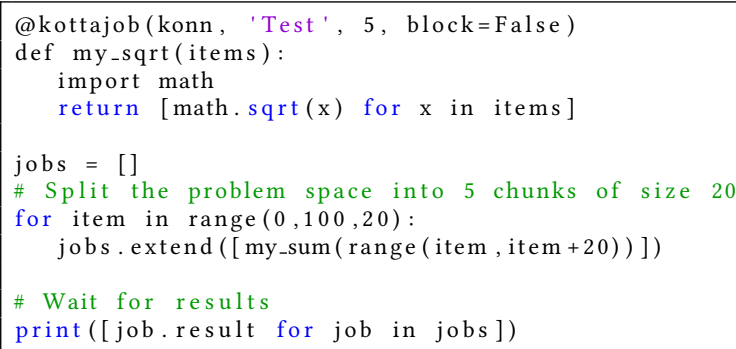

Since the function my_sqrt ( ) is decorated with the option block=Fals calls to the function return immediately with a future. A future is a common term used to describe an object that acts as a proxy for which a value is not yet known. The advantage of this approach is that users may execute several such functions, all of which are executed in parallel.

Decoratated functions also take special keywords inputs and outputs which are used to indicate to Cloud Kotta that special (protected) datasets must be staged-in or staged-out. In this case, Cloud Kotta uses its secure data enclave to locate the requested dataset. Cloud Kotta then invokes its standard security mechanisms to authorize access to the data if the user has privileges to access the dataset. Since each task is handled by Cloud Kotta there's an auditable record of every compute task that touched protected datasets.

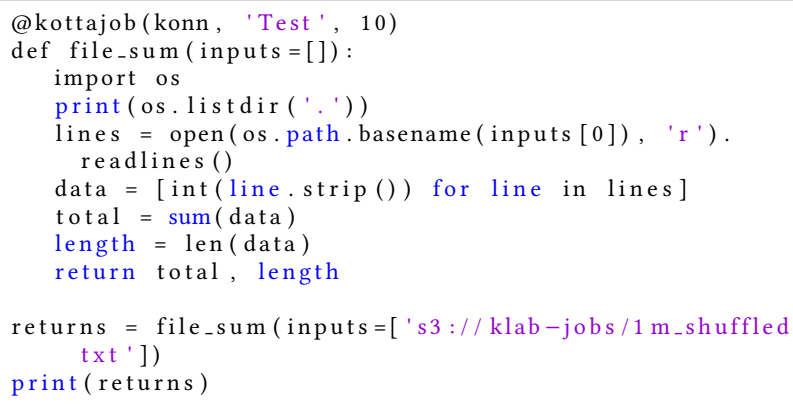

The kot ta library by default captures both STDOUT and STDERR streams. These are accessible through the future returned from a non-blocking call, or when a blocking call fails with an exception. This simplifies debugging during the development process. It is also important to note that Cloud Kotta logs the entirety of the task allowing both retrospective and prospective provenance. For tasks running longer than 5 minutes Cloud Kotta also captures continuous CPU and Memory utilization information that can be viewed through the Web GUI. The @kottajob decorator also takes a requirements keyword argument that accepts a specially formatted string. This allows for installing and setting up requisite python packages before the decorated function is executed on the compute nodes.

Finally, the library also supports specifying execution of arbitrary applications in a batch model. This allows the user to easily plug in existing applications written in any language and to subsequently manage these applications via Python. This ensures that complex workflows with unusual requirements such as ensuring that no data is fetched to the Jupyter notebook are possible. Here is a minimal example:

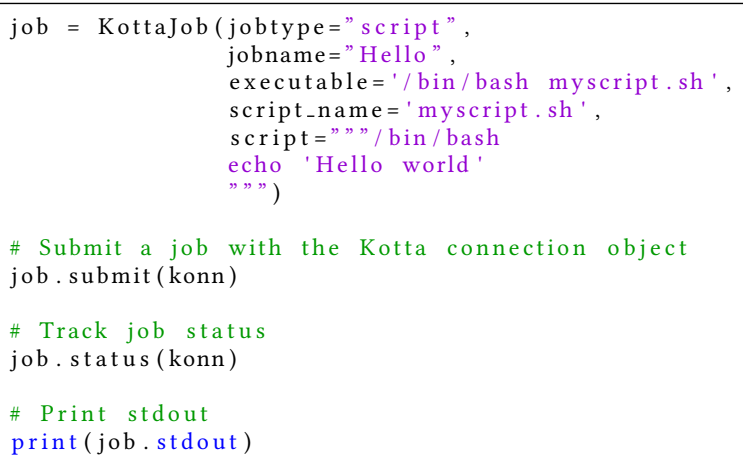

\section{APPLICATIONS}

Adding interactivity to Cloud Kotta for rapid ideation and iteration was motivated by a gap between the functionality of the computational environment and the type of interrogative, discovery science adopted by researchers. This gap was particularly noticeable in 
work being carried out by Knowledge Lab researchers on the Clarivate Analytics (formerly the IP \& Science business of Thomson Reuters) Web of Science and the ITHAKA FSTOR datasets. This work, situated in the Computational Social Sciences and the broader Science of Science, seeks to understand the institutions and dynamics of knowledge production by modeling the scientific system from publication data [9]. In what follows, we outline three concrete use cases that initially motivated and then benefited from our extension of Cloud Kotta.

Cloud Kotta has been used to analyze the full Web of Science raw data and to compute two measures of the scientific impact of individual journals and individual papers - Eigenfactor and the Article Influence metrics, respectively [8]. The general analysis pipeline is as follows. The Web of Science data files are converted from their raw XML format to a structured collection of lists and dictionaries for each year. From this intermediate form, the data are processed and aggregated in batches corresponding to moving window years (e.g., as specified by the Eigenfactor computation). Of course, any large computational infrastructure would have proved efficacious for this job. However, because of the sensitive nature of the data and accompanying access agreements, the use of the Cloud Kotta secure data enclave and computational infrastructure was necessitated. The ability to collocate propriatery data and compute within the same virtual private and secure cloud was also convenient, due to the size of the dataset ( $100 \mathrm{~Gb}$ ), the compute time (several hours per job), and the susceptibility of the task to parallelization (the initial dataset is split into year-labeled files). The collection of jobs was submitted en masse through the Cloud Kotta Client Python interface described in this paper and outputs were fetched in a similar manner. It is worth noting that, while the selection of machine types and OS images are hidden from the user, the system is flexible enough that, upon request by the user, instances with, for example, more memory or an image with task specific pre-installed libraries, can be made available -which is what occurred in this case. For future bibliometric work, users will be in a position to make minor modifications to the setup and codebase used in the Eigenfactor computation thanks to Cloud Kotta's job publication features.

In addition to parsing tens of millions of files and calculating metrics from extracted metadata, the interactive Jupyer analysis environment is ideal for generating and then iteratively interrogating networks (e.g. graphs) comprised of billions of links. Much of the work that goes into the initial analysis of networks is rather exploratory. However, when a network is as massive as, for instance, the citation network that underlies the scientific enterprise, rapid ideation is stymied by secure but rigid computational environments with queue based job submission protocols.

In order to get a better sense of the citation network in the Web of Science, researchers needed to parse tens of millions of raw XML files, generate an edge list of all source papers and their respective citations targets, and create an adjacency list. The goal was to make it easier to directly parse, interrogate, and derive summary statistics about citation counts for any specific paper in the Web of Science. The derived, proprietary edge list citation network is $20 \mathrm{~GB}$ uncompressed, making it immediately a memory and disk-intensive problem for local machines or small, single nodes. Moreover, because the data is proprietary and subject to data use agreements, the network cannot be replicated and processed locally. Given that we are working with billions of citations and parallelizable algorithms for assembling the adjacency list, it makes economic and legal sense to utilize the Kotta decorator and farm the problem out to large nodes. Moreover, once the graph has been generated, basic summary statistics can be fetched directly from the Jupyter notebook by running simple network analysis functions with the Cloud Kotta decorator. In this way, even a network with billions of links can be explored in the straightforward and intuitive way that researchers have come to expect in an area of discovery science.

Finally, users have found the new, interactive analysis capabilities of Cloud Kotta to be useful in conducting research using natural language processing and text mining on big, scholarly, data. For instance, in one project, researchers seek to analyze the rhetorical framing of Adam Smith and his political philosophy from The Wealth of Nations. In order to do this, users first conducted text mining to identify terms and concepts related to Adam Smith as well as the contexts in which they appear within the body of social science literature from 1890s to the 2010s. Cloud Kotta allowed researchers to actively and iteratively filter through candidate terms and concepts and relate them to similar concepts and phrases found in Adam Smith's work. This problem required leveraging $20 \mathrm{~GB}$ of protected OCR'd (optical character recognition) data from JSTOR, and performing relatively expensive analyses (e.g., tf-idf, word2vec, lda, etc.) which makes the problem just out of reach for many personal, local machines, both in terms of memory and disk, as well as data use agreements.

Of course, it was possible to carry out many of the tasks required of these projects in more traditional, queue based compute environments. Several of the tasks, however, could not have been performed on more traditional data enclaves, as these have tended to be local, relatively small, and isolated machines. Nevertheless, enabling researchers to rapidly ideate and iterate on code and analysis has greatly improved the research process in each of the applications and has, as a result, sped discovery.

\section{RELATED WORK}

There are few systems that enable secure management and analysis of research data. While researchers have explored methods for enhancing client-side software for analysis of large amounts of local data [13] and exploited hybrid cloud models to scale analytics [5], they do not provide a flexible, integrated environment for managing and analyzing data, and to the best of our knowledge none support interactive analysis on protected data. Perhaps the most similar approach to ours is the data capsule [17] model used by the Hathi Trust to support secure, non-consumptive analysis of data by leveraging controlled virtual machines. However, this model is designed to use virtual machine constructs and therefore it is lower level and does not support interactive analytics via an easy to use Jupyter notebook.

Science gateways [16] have long been used to provide simplified, domain-specific access to large scale computing infrastructure. However, unlike Cloud Kotta, they focus primarily on HPC infrastructure and typically support fixed analysis types (via a web form) and queue-based execution models. While some gateways now leverage cloud infrastructure [11] none provide the rich security 
policies, extensible execution model, or interactive analysis model provided by Cloud Kotta.

There are several efforts to provide multi-user, interactive analysis environments built around Jupyter notebooks. For example, JupyterHub [3], the system we build upon here, allows multiple users to instantiate instances of Jupyter notebooks. It is typically deployed on a large machine and uses a proxy-based model to forward requests to a particular Jupyter instance. Tmpnb [4] aims to satisfy a similar multi-user model for running temporary notebooks. It launches Docker containers for each notebook and proxies requests to each container. Tmpnb has been used to provide temporary notebooks for replicating analyses published in Nature [14]. Binder [1] applies a similar model, using Docker containers to execute Jupyter notebooks directly from GitHub repositories. The public deployment is hosted on a small Google Compute Engine Cluster that can scale with usage. While these systems provide on-demand interactive analysis environments, they do so at the notebook level and focus on computational reproducibility. Cloud Kotta instead offers a similar environment for composing and executing notebooks that analyze protected data. Our model is unique in that it extends these notebook environments via language constructs to exploit access to secure data and use of large scale computing resources.

Finally, there are many examples of libraries and programming languages that aim to simplify the use of parallel and distributed computing resources. In particular, IPythonParallel provides a simple model for enabling parallel execution of Python functions. IPythonParallel allows user-defined decorators to be associated with functions which are subsequently sent as parallel jobs to a predefined execution system. This model provides no support for data management, implementing security models at the granularity of functions, or autoscaling in a cloud environment. It would require considerable effort to integrate such frameworks with the Cloud Kotta security fabric.

\section{SUMMARY}

We have described the enhancements we have made to the Cloud Kotta secure data enclave to support interactive data analytics on protected data. The motivation for our work was based on the needs of an increasingly common class of researcher: those who utilize exploratory data science to analyze large, protected datasets. To address the needs of these researchers we described how we have integrated Jupyter notebooks with Cloud Kotta to fulfill the significant gap between interactive and queue-based systems. Our approach relies on JupyterHub to enable multi-user Jupyter environments and the creation of a lightweight Python library that supports semi-transparent execution of code functions using a Python decorator. Initial experiences with this platform have been positive, several researchers have now adopted this system in their every-day research.

Our future work is primarily focused on completing the integration of our authentication and authorization systems. In so doing, we will simplify user experience by enabling them to use the same identities in both environments and by transparently enabling connections from Jupyter notebooks to Cloud Kotta. As a secondary goal we will extend the kotta library to include support for dependency management. This support will allow users of our system to compose more complex workflows comprised of independent steps.

\section{ACKNOWLEDGMENTS}

The authors would like to thank Nathan Bartley and Alexander Belikov for testing the system in its early stages of development.

\section{REFERENCES}

[1] 2017. Binder. (2017). http://mybinder.org//[lastaccessed,March2017

[2] 2017. Jupyter Notebook. (2017). http://jupyter.org/[lastaccessed,March2017

[3] 2017. JupyterHub. (2017). https://github.com/jupyterhub/ jupyterhub[lastaccessed, March2017

[4] 2017. tmpnb, the temporary notebook service. (2017). https://github.com/jupyter/ tmpnb[lastaccessed,March2017

[5] S. Abramson, W. Horka, and L. Wisniewski. 2014. A Hybrid Cloud Architecture for a Social Science Research Computing Data Center. In Proceedings of the 34th International Conference on Distributed Computing Systems Workshops (ICDCSW). 45-50. DOI : http://dx.doi.org/10.1109/ICDCSW.2014.32

[6] Y. N. Babuji, K. Chard, A. Gerow, and E. Duede. 2016. Cloud Kotta: Enabling secure and scalable data analytics in the cloud. In IEEE International Conference on Big Data (Big Data). 302-310. DOI : http://dx.doi.org/10.1109/BigData.2016. 7840616

[7] Y. N. Babuji, K. Chard, A. Gerow, and E. Duede. 2016. A secure data enclave and analytics platform for social scientists. In 12th IEEE International Conference on e-Science (e-Science). 337-342. DOI : http://dx.doi.org/10.1109/eScience.2016. 7870917

[8] Carl T Bergstrom, Jevin D West, and Marc A Wiseman. 2008. The Eigenfactorffl metrics. Journal of Neuroscience 28, 45 (2008), 11433-11434.

[9] James A Evans and Jacob G Foster. 2011. Metaknowledge. Science 331, 6018 (2011), 721-725.

[10] Jacob G Foster, Andrey Rzhetsky, and James A Evans. 2015. Tradition and innovation in scientistsfi research strategies. American Sociological Review 80, 5 (2015), 875-908.

[11] Ravi Madduri, Kyle Chard, Ryan Chard, Lukasz Lacinski, Alex Rodriguez, Dinanath Sulakhe, David Kelly, Utpal Dave, and Ian Foster. 2015. The Globus Galaxies platform: delivering science gateways as a service. Concurrency and Computation: Practice and Experience 27, 16 (2015), 4344-4360. DOI: http://dx.doi.org/10.1002/cpe.3486

[12] Daniel A. Reed and Jack Dongarra. 2015. Exascale Computing and Big Data. Commun. ACM 58, 7 (June 2015), 56-68. DOI : http://dx.doi.org/10.1145/2699414

[13] M. A. Saleem, B. Varghese and A. Barker. 2014. BigExcel: A web-based framework for exploring big data in social sciences. In Proceedings of the IEEE International Conference on Big Data (Big Data). 84-91. DOI : http://dx.doi.org/10.1109/BigData. 2014.7004458

[14] Helen Shen. 2014. Interactive notebooks: Sharing the code. Nature 515, 7525 (2014), 151-152.

[15] Arthur W Toga, Ian Foster, Carl Kesselman, Ravi Madduri, Kyle Chard, Eric W Deutsch, Nathan D Price, Gustavo Glusman, Benjamin D Heavner, Ivo D Dinov, Joseph Ames, John Van Horn, Roger Kramer, and Leroy Hood. 2015. Big biomedical data as the key resource for discovery science. Fournal of the American Medical Informatics Association 22, 6 (2015), 1126. DOI:http: //dx.doi.org/10.1093/jamia/ocv077

[16] Nancy Wilkins-Diehr. 2007. Special Issue: Science Gatewaysfi!?Common Community Interfaces to Grid Resources. Concurrency and Computation: Practice and Experience 19, 6 (2007), 743-749. DOI : http://dx.doi.org/10.1002/cpe.1098

[17] Jiaan Zeng, Guangchen Ruan, Alexander Crowell, Atul Prakash, and Beth Plale. 2014. Cloud Computing Data Capsules for Non-consumptiveuse of Texts. In Proceedings of the 5th ACM Workshop on Scientific Cloud Computing (ScienceCloud). ACM, 9-16. DOI : http://dx.doi.org/10.1145/2608029.2608031 